Journal of

\title{
Dynamics of paramagnetic nanostructured rods under rotating field
}

\author{
B. Frka-Petesic ${ }^{\mathrm{a}}$, K. Erglis ${ }^{\mathrm{b}}$, J.F. Berret ${ }^{\mathrm{c}}$, A. Cebers ${ }^{\mathrm{b}}$, V. Dupuis ${ }^{\mathrm{a}}$, J. Fresnais ${ }^{\mathrm{a}}$, R. Perzynski ${ }^{\mathrm{a}, *}$, O. Sandre ${ }^{\mathrm{a}}$ \\ ${ }^{a}$ UPMC Univ. Paris 6, Laboratoire PECSA - UMR 7195 UPMC-CNRS-ESPCI, Case 514 Place Jussieu, F-75005 Paris FRANCE \\ ${ }^{b}$ Depatment of Theoretical Physics, University of Latvia, Zellu 8, Riga, LV-1002 LATVIA \\ ${ }^{c}$ Lab. MSC - UMR 7057 CNRS - Univ. Paris Diderot, Bât. Condorcet 10 rue Alice Domon et Léonie Duquet, F-75205 Paris Cedex 13 FRANCE
}

Author manuscript version of article available in its final form on the publisher's website: DOI 10.1016/j.jmmm.2010.11.036

\begin{abstract}
The dynamical rotational behavior of magnetic nanostructured rods based on the auto-association of maghemite nanoparticles and block-copolymers is probed by optical microscopy under rotating fields in a simple 1 iquid. The re-orientation of the rods by a field rotated by $90^{\circ}$ is first studied. The measured relaxation is characteristic of paramagnetic objects. Under a stationnary rotating field, a synchronous rotational regime is observed at low field frequency. Above a frequency threshold which scales as $H^{2}$, the dynamics becomes asynchronous with back-and-forth rotations. These behaviors are well predicted by the presented model.
\end{abstract}

PACS 82.70.Dd; 81.07-b; 83.80.Uv; 81.16.Dn

Keywords: Colloids; Nanocomposites; Block copolymers; Self-assembly

\section{Introduction}

Probing the local rheology of complex media, such as the internal compartments of a living cell [1], is a current stake of research. Magnetic rods gives the opportunity of such a local probing under a time-dependent field. Direct optical measurements are possible with sub-millimetric rods $[2,3]$ and even at smaller scales, thanks to the under-field chaining of sub-micronic magnetic particles [4]. Such particles joined together in a chain through a temporary or a permanent link, can be used for mixing chemical moities in microfluidic devices [5] and allows a local rheological probing in the linear hydrodynamic regime $[1,6,7]$. However for large aspect ratios, these softly-linked chains present shape instabilities $[4,8]$ analogous to the one observed with magnetic liquid needles [9]. Rigid magnetic rods would not present such limitations.

*Corresponding author. Tel.: 0033-1-444273267; fax: 0033-1444273228.

Email address: regine.perzynski@upmc.fr (R. Perzynski)
We present here a study by optical microscopy, of the rotational behavior in a simple liquid under an applied rotating field, of rigid magnetic nanostructured rods, obtained by the co-association of magnetic nanoparticles and di-block copolymers $[10,11]$. We first probe the system under a field abruptly rocked by $90^{\circ}$ and study the rotation of the rods as a function of field amplitude $H$. We then analyze the rotational behaviour of these rods under an applied field rotating at angular frequency $\omega$ as a function of $H$ and $\omega$, both in the linear regime and in the non-linear one, following the descriptions presented in [12].

\section{Synthesis and shape characteristics of the micro- rods}

The magnetic nanostructured rods result from the under-field auto-association of maghemite nanoparticles synthesized following Massart's general procedure [13] and block co-polymers denoted as PTEA $11 k^{-}$ b-PAM ${ }_{30 k}$ provided by Rhodia [14]. PTEA stands for poly(dimethyl ammonium ethylacrylate methyl sulfate) and is positively charged, while PAM stands for 
poly(acrylamide) and is neutral. PTEA blocks glue together the negatively charged nanoparticles and PAM blocks provide the rods with a neutral corona that takes the rods apart from each other. The process followed for the synthesis of the rods is detailed in [10]. The maghemite nanoparticles (NP's) used here are coated with $2000 \mathrm{~g} / \mathrm{mol}$ PAA poly(acrylic acid). The distribution of magnetic NP diameters is well described by a log-normal distribution with a mean diameter 7.1 $\mathrm{nm}$ and a polydispersity 0.26 . These superparamagnetic NP's have a dipolar magnetic interaction parameter $3 \chi_{0} / 24 \Phi=0.48$ where $\chi_{0}$ is the initial magnetic susceptibility of a liquid dispersion of these nanoparticles at low volume fraction $\Phi$ (see [15]). Transmission Electron Microscopy (TEM) reveals the elongated shape of the obtained clusters, as illustrated in Figure 1. The observation by optical microscopy of dilute aqueous dispersions of these rods allows to determine their distribution of lengths $L$, as given by the inset of Fig. 1.

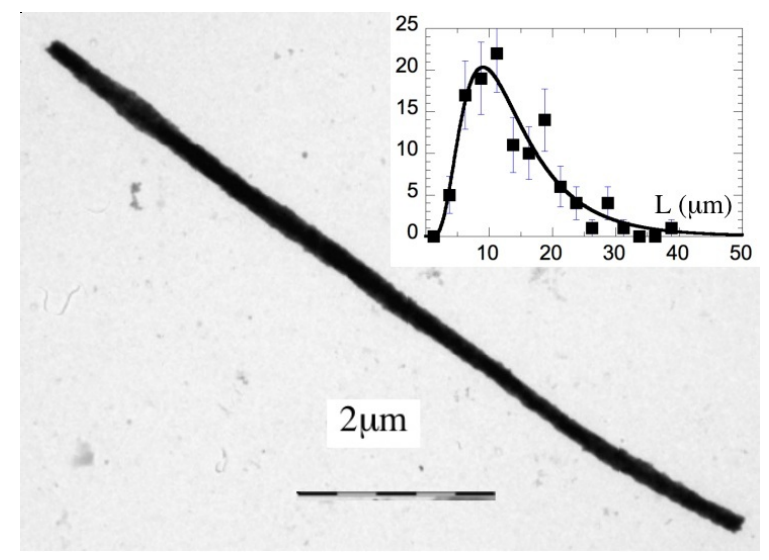

Figure 1: TEM picture of a nanostructured rod. Inset : Distribution of length $L$ of the rods in a dilute aqueous dispersion as determined by optical microscopy. Full line : fit by a log-normal distribution with median length $12.3 \mu \mathrm{m}$ and polydispersity 0.5

\section{Experimental set-up}

For the dynamical study under rotating field, dilute dispersions of magnetic rods are placed between two glass slides separated by $0.03 \mathrm{~mm}$ double-sided sticky tape with an aperture cut in the center. The rotating magnetic field is created by four water-cooled coils giving a field $0-20 \mathrm{kA} / \mathrm{m}$ in the frequency range $0.1-5$ $\mathrm{Hz}$. The rotation of the filaments is captured by a Zeiss Universal microscope and a JAI CV-S3200 video camera. With time, the rods slowly sediment under gravity and sometimes stick to the cell wall by one end. In the experiment which duration is short with respect to the sedimentation time, we retain only the rods which are freely rotating in the liquid carrier. The registered images are analyzed with the Image J code [17] .

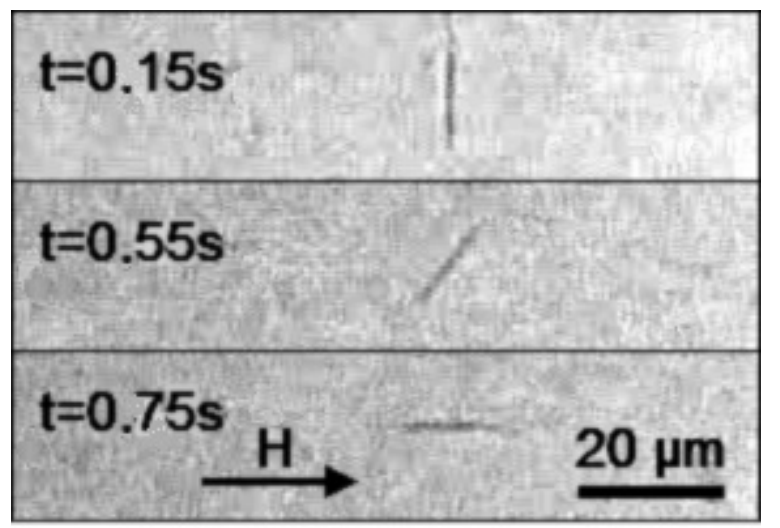

Figure 2: Re-orientation of a rod with $L=15 \mu \mathrm{m}$ under a field of amplitude $H=10 \mathrm{kA} / \mathrm{m}$ abruptly rotated by $90^{\circ}$ and observed as a function of time by optical microscopy.

\section{Forced re-orientation under a rocking field}

Experiments of rod re-orientations under a static applied field, abruptly rotated by $90^{\circ}$ are first performed. The rods follow the field orientation, as attested by Figure 2 with a rod of length $15 \mu \mathrm{m}$. The angle $\beta$ between the field and the rod directions is registered as a function of time $t$ in Figure 3. The inflexion point of the relaxation $\beta=f(t)$ at $\beta=45^{\circ}$ confirms the paramagnetic nature of the rods as observed in $[10,11]$.

\section{Theoretical background}

The paramagnetic rod submitted to a magnetic field $\vec{H}=H_{0} \vec{h}$ has a magnetic moment $\vec{\mu}$ proportional to its magnetic susceptibility tensor $\overline{\bar{\chi}}: \vec{\mu}=\mu_{0} \overline{\bar{\chi}} V_{M} \vec{H}$ where $V_{M}$ is the magnetic volume of the rod. Projecting $\vec{\mu}$ along its main axes $\vec{e}_{\|}$and $\vec{e}_{\perp}$ and with $\beta=\left(\vec{h}, \vec{e}_{\|}\right)$, it writes :

$$
\vec{\mu}=\chi^{\|} V_{M} H_{0} \cos \beta \vec{e}_{\|}+\chi^{\perp} V_{M} H_{0} \sin \beta \vec{e}_{\perp}
$$

the anisotropy of susceptibility of the rod being $\Delta \chi=$ $\chi^{\|}-\chi^{\perp}$. If the magnetic field $\vec{H}$ rotates (abruptly or at 
the pulsation $\omega)$ in the plane $\left(\vec{e}_{x}, \vec{e}_{y}\right)$, the rod experiences a magnetic torque $\vec{\Gamma}_{\text {magn }}$ :

$$
\vec{\Gamma}_{\text {magn }}=\vec{\mu} \times \vec{H}=\frac{\mu_{0} \Delta \chi V_{M} H_{0}^{2}}{2} \sin 2 \beta \vec{e}_{z}
$$

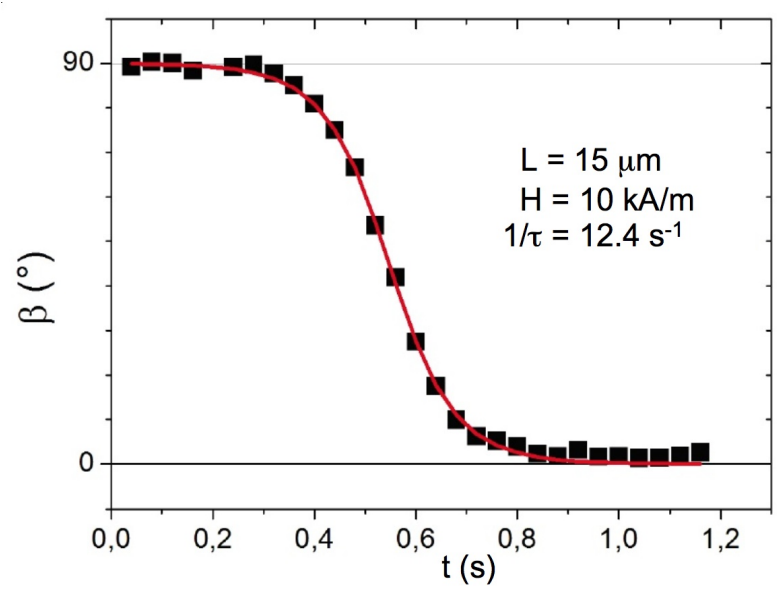

Figure 3: Relaxation as a function of time $t$ of the angle $\beta$ between the field and the rod directions - same experimental conditions as in Figure 2. The full line is a best fit by expression (5).

and a viscous torque $\vec{\Gamma}_{\text {visc }}[16]$ :

$$
\vec{\Gamma}_{v i s c}=-f_{\eta} \vec{\Omega}=-\frac{\pi \eta L^{3}}{3 \ln (L / D)-2} \vec{\Omega}
$$

where $f_{\eta}$ is the coefficient of hydrodynamic drag experienced by the rod, $\vec{\Omega}$ the instantaneous angular velocity of the rod, $\eta$ the viscosity of the fluid carrier and $D$ the diameter of the rod section.

\subsection{Field abruptly rotated by $90^{\circ}$}

If the re-orientation of the rod is forced under a quick rocking at right angle of the field direction, the relaxation is then driven by the following differential equation :

$$
\frac{\mathrm{d} \beta}{\mathrm{d} t}=-\frac{1}{2 \tau} \sin 2 \beta \quad \text { with } \quad \frac{1}{\tau}=\frac{\mu_{0} \Delta \chi V_{M} H_{0}^{2}}{f_{\eta}}
$$

The equation has three solutions $(\bmod \pi): \beta=0($ stable equilibrium), $\beta=\pi / 2$ (unstable equilibrium) and :

$$
\beta(t)=\arctan \left[\exp \left(-\frac{t-t_{\pi / 4}}{\tau}\right)\right]
$$

where $t_{\pi / 4}$ is defined as $\beta\left(t=t_{\pi / 4}\right)=\pi / 4$.

\subsection{Field rotating at angular frequency $\omega$}

In the experiment under a field rotating at angular frequency $\omega$, the angle $\theta=\left(\vec{e}_{\|}, \vec{e}_{x}\right)$ between the rod and the axis $\vec{e}_{x}$ is equal to :

$$
\theta=\omega t-\beta
$$

It leads to the differential equation in $\beta$ :

$$
\frac{\mathrm{d} \beta}{\mathrm{d} t}=\omega-\omega_{c} \sin 2 \beta \quad \text { with } \quad \omega_{c}=\frac{\mu_{0} \Delta \chi V_{M} H_{0}^{2}}{2 f_{\eta}}
$$

where the critical pulsation $\omega_{c}$ is introduced.

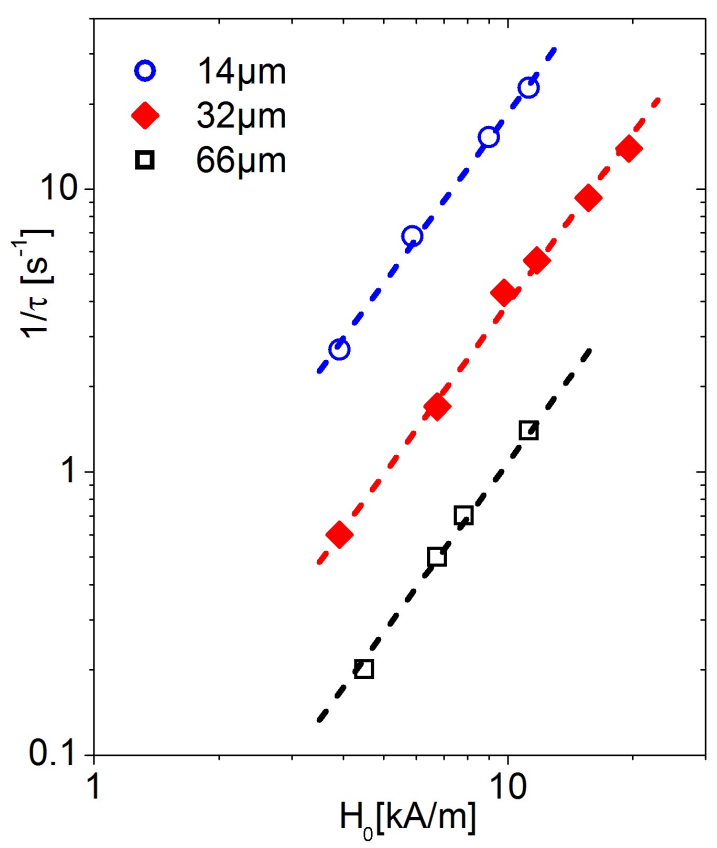

Figure 4: Inverse of the relaxation time $\tau$ of various rods, measured under a field abruptly rotated by $90^{\circ}$ and plotted as a function of field amplitude $H_{0}$; Various symbols correspond to various rod lengths $L$. Dashed lines are guides for the eye of slope 2.

This equation has two solutions $[18,12,19,20]$ :

- for $\omega<\omega_{c}$, there is a stationary solution in $\beta$ : $\sin 2 \beta=\omega / \omega_{c}$ and thus

$$
\beta=\frac{1}{2} \arcsin \left(\frac{\omega}{\omega_{c}}\right)
$$

The expression of $\theta(t)$ is then affine :

$$
\theta(t)=\omega t-\frac{1}{2} \arcsin \left(\frac{\omega}{\omega_{c}}\right)
$$


- for $\omega>\omega_{c}$, there is a periodic solution for $\beta$, and pseudo-periodic for $\theta$.

$$
\begin{aligned}
\beta(t) & =\arctan \left[\frac{\omega_{c}}{\omega}\right. \\
+ & \left.\sqrt{1-\left(\frac{\omega_{c}}{\omega}\right)^{2}} \tan \left(\sqrt{1-\left(\frac{\omega_{c}}{\omega}\right)^{2}} \omega\left(t-t_{0}\right)\right)\right]
\end{aligned}
$$

The mean angular velocity $\bar{\Omega}=\langle\dot{\theta}\rangle$ of the rod, taken over a period of time long with respect to $1 / \omega_{c}$, then writes as $\bar{\Omega}=\omega-\sqrt{\omega^{2}-\omega_{c}^{2}}$ [19].

\section{Experimental results}

\subsection{Field abruptly rotated by $90^{\circ}$}

The $\beta(t)$ relaxation under forced re-orientation after a quick rocking at right angle of the field direction is nicely reproduced by expression (5). An illustration of the good fit quality is given in Figure 3. Relaxation times $\tau$ measured for several rods are collected in Figure 4 . At the first order $1 / \tau$ scales as $H^{2}$ as predicted by expression (5). We should remark that if the field is not exactly rocked at $\theta_{\text {init }}=90^{\circ}$, but at a smaller angle $\theta_{\text {init }}$, it is however possible to nicely determine the relaxation time $\tau$.

\subsection{Field rotating at angular frequency $\omega$}

Indeed with a field rotating at constant $\omega$, the two rotational regimes are observed experimentally. Below a critical pulsation $\omega_{c}$, scaling as $H_{0}^{2}$, each micro-rod synchronously follows the applied field with a phase-lag $\beta$ (see Inset of Figure 5) as also observed in [16]. As the pulsation $\omega$ of the magnetic field increases, the angular velocity $\Omega$ of the rod increases (see main part of Figure 5) up to $\omega=\omega_{c}$, for which the phase-lag $\beta$ reaches the value $\pi / 4$. Then the rotational dynamics of the $\operatorname{rod}$ becomes jerky, with back-and-forth rotations more and more pronounced as $\omega$ increases. As shown in Figure 5, the mean angular velocity of the $\operatorname{rod} \bar{\Omega}$ then decreases towards zero.

Polar representations, such as that of Figure 6, plotting the time $t$ as radial coordinate and the angle $\theta$ as polar angle, clearly distinguish the two regimes. At low frequencies, the synchronous regime leads to Archimedes spirals while for $\omega>\omega_{c}$ some back-andforth rotations mark the jerky regime, they are more and more numerous as the pulsation increases above $\omega_{c}$.

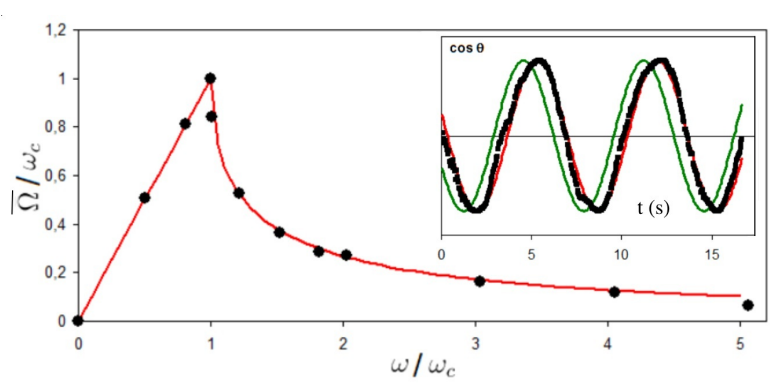

Figure 5: Reduced representation of the mean angular velocity $\bar{\Omega}$ of a rod of length $L=10 \mu \mathrm{m}$ as a function of the pulsation $\omega$ of the field normalized at the limit of the synchronous regime where $\omega=\omega_{c}\left(H_{0}\right.$ $=8 \mathrm{kA} / \mathrm{m}$ and $\left.\omega_{c}=6.2 \mathrm{rad} / \mathrm{s}\right)$. Inset : temporal evolution of the rod orientation ( $\cos \theta$ - thick line) compared to the orientation of the field (thin line) for a rod of length $L=14 \mu \mathrm{m}$ under a field amplitude $H_{0}=$ $3.2 \mathrm{kA} / \mathrm{m}$ at $\omega=0.94 \mathrm{rad} / \mathrm{s}$ just below $\omega_{c}=0.95 \mathrm{rad} / \mathrm{s}$.
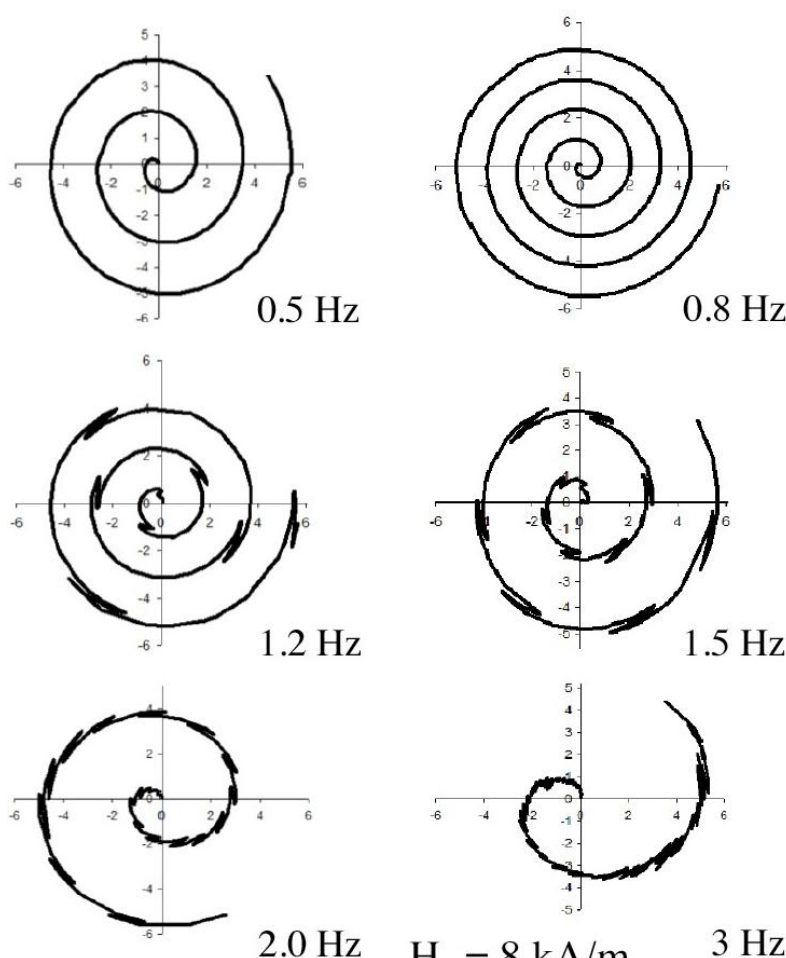

$\mathrm{H}_{0}=8 \mathrm{kA} / \mathrm{m}$

$3 \mathrm{~Hz}$

Figure 6: Polar representation $\theta(\mathrm{t})(\theta$ polar angle; time $t$ radial coordinate) of the rotational dynamics of the same rod as in Figure 5 with $L=10 \mu \mathrm{m}$ under a magnetic field of amplitude $H_{0}=8 \mathrm{kA} / \mathrm{m}$ rotating at various frequencies (from top to bottom: left $\omega=3.1,7.5$ and 12.6 $\mathrm{rad} / \mathrm{s} ;$ right $\omega=5.0,9.4$ and $18.8 \mathrm{rad} / \mathrm{s})$; here $\omega_{c}=6.2 \mathrm{rad} / \mathrm{s}$ 


\section{Discussion}

Identifying hydrodynamic and magnetic characteristics of the rods, we assume that the rod magnetic volume can be writen as :

$$
V_{M}=\frac{\pi L D^{2}}{4}
$$

Following expressions (3) and (4), we can rewrite the inverse relaxation time of forced re-orientation as :

$$
\frac{1}{\tau}=\frac{\mu_{0} \Delta \chi \tilde{H}^{2}}{4 \eta}
$$

where

$$
\tilde{H}=H_{0} \frac{D}{L} \sqrt{3 \ln \frac{L}{D}-2}
$$

Figure 7 presents the results of Figure 4, re-plotted as a function of $\tilde{H}$ which is calculated with $D=150$ $\mathrm{nm}$, a value compatible with TEM observations (optical images have not enough accuracy for a precise determination of $D$ ). A master curve of slope 2 is then obtained in Figure 7, leading to a value the anisotropy of susceptibility $\Delta \chi=\chi^{2} /(2+\chi)=0.26$ thus a susceptibility of the internal material $\chi=0.87$.

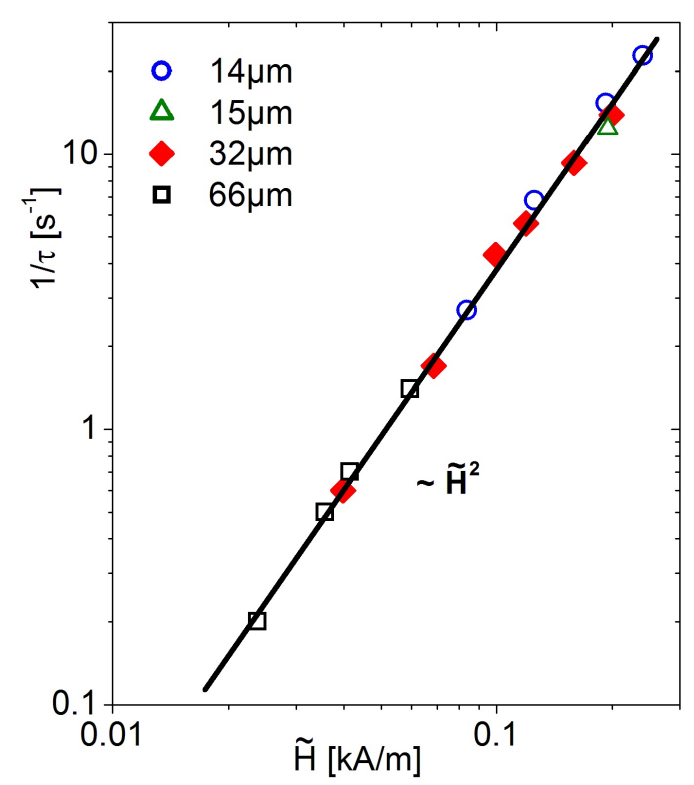

Figure 7: Inverse of the relaxation time $\tau$ of various rods, measured under a field abruptly rotated by $90^{\circ}$ and plotted as a function of the reduced variable $\tilde{H}$ given by expression (13). The line is an adjustment of the data by expression (12) with $D=150 \mathrm{~nm}$.
It is also possible to deduce the rod anisotropy of susceptibility $\Delta \chi$ from the experimental threshold $\omega_{c}$ using expressions (3) and (7). Figure 8 demonstrates for a particular rod of length $L=14 \mu \mathrm{m}$ the pretty good agreement between the field-dependence of $2 \omega_{c}$ and of $1 / \tau$ deduced from the two kinds of measurements. From these determinations, we find $\Delta \chi=0.26$ (resp. 0.16) for $D=150 \mathrm{~nm}$ (resp. $200 \mathrm{~nm}$ ), thus a susceptibility of the internal material $\chi=0.87$ (resp. 0.65). Taking in account the low value of the dipolar magnetic interaction parameter of the present superparamagnetic nanoparticles, this internal susceptibility is here directly proportional to the mean internal concentration $\Phi_{\text {int }}$ of nanoparticles inside the rods [15]. With $D=150 \mathrm{~nm}$ (resp. $200 \mathrm{~nm}$ ) we find $\Phi_{i n t}=23 \%$ (resp. 17\%) of the same order as in [10].

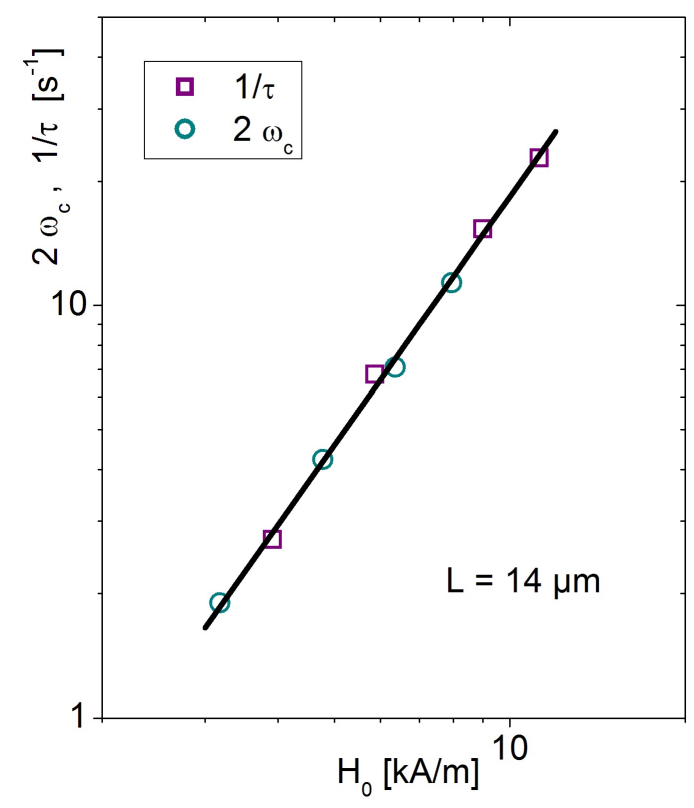

Figure 8: $\log -\log$ representation of $2 \omega_{c}$ as a function of the amplitude of the rotational field $H_{0}$ for a rod of length $L=14 \mu \mathrm{m}$, compared to $1 / \tau$ determined under a field abruptly rotated by $90^{\circ}$ and plotted as a function of field amplitude $H_{0}$.

If now a small ellipticity of the rotating field is introduced, it sensibly modifies the shape of the curve $\bar{\Omega}=$ $\mathrm{f}(\omega)$ of Figure 5, as predicted in [19], slightly reducing the experimental value of $\omega_{c}$ while keeping its $H^{2}$ dependence. This behaviour will be detailled elsewhere. A remarquable point is that it introduces an anisotropy in the back and forth motion of the rods as it is here illustrated by the polar representation $\theta(\mathrm{t})$ of Figure 9 for the same rod as in Figure 6 with $L=10 \mu m$. 


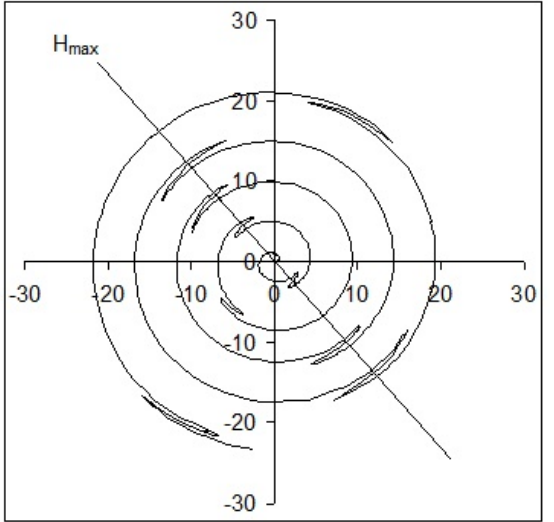

Figure 9: Polar representation $\theta(\mathrm{t})$ of the rotational dynamics of a rod with $L=10 \mu \mathrm{m}$ under a magnetic field slightly elliptic of mean amplitude $H_{\max }=4.8 \mathrm{kA} / \mathrm{m}$ rotating at $\omega=2.5 \mathrm{rad} / \mathrm{s}$ (field ellipticity $H_{\min } / H_{\max }$ of the order of 0.87 ). The induced back and forth motion is anisotropic.

At high frequency, this anisotropy allows to observe localized motions of the rod around the direction $H_{\max }$ as predicted in [19] (see Fig. 10).

\section{Conclusion}

Probing the rotational dynamics of our rigid magnetic rods, dispersed in a standard liquid, either by reorienation along field rocked at $90^{\circ}$ or by a steady rotating field, we demonstrate the paramagnetic nature of the rods and characterize their anisotropic susceptibility. The behaviour under steady rotating field fairly well follows in the linear and in the non-linear regime the theoretical predictions initially developped - for pairs of magnetic holes in a ferrofluid in $[19,20]$ and - for paramagnetic ellipsoids in [12]. In [12], paramagnetic ellipsoids of aspect ratio up to 5 were tested, our magnetic rods have an aspect ratio more than 10 times larger.

These magnetic rods will thus find applications as active elements in microfluidic devices viz. mechanical stirrers or pump elements. A rheological probing of complex medium has now to be undertaken with these magnetic nanostructured rods.

\section{Acknowledgments}

This work was supported by ANR "ITC-Nanoprobe", grant of University of Latvia ${ }^{\circ}$ ESS2009/6, Féd. F21 UPMC-Paris6, programs ECONET $n^{\circ}$ 16274QL and OSMOSE $n^{\circ}$ 22497YE. We thank Aude Michel (PECSA) for the TEM pictures.

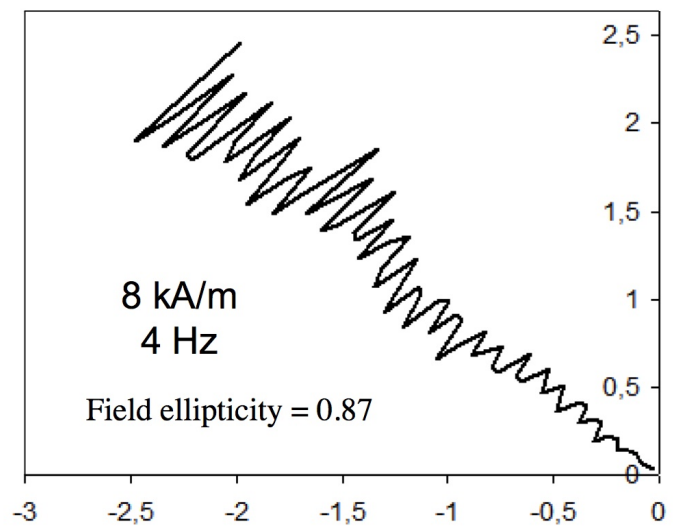

Figure 10: Polar representation of $\theta(t)$ of a localized mode of a rod with $L=10 \mu m$, observed in an experiment with a field ellipticity $H_{\text {min }} / H_{\text {max }}$ of the order of $0.87\left(H_{\text {max }}=8 \mathrm{kA} / \mathrm{m}\right.$ and $\left.\omega=4 \mathrm{~Hz}\right)$.

\section{References}

[1] C. Wilhelm Phys. Rev. Lett. 101 (2008) 028101.

[2] C. Wilhelm, F. Elias, J. Browaeys, A. Ponton and J.C. Bacri Phys. Rev. E 66 (2002) 021502.

[3] D. Letellier, O. Sandre, C. Ménager, V. Cabuil and M. Lavergne Mat. Sci. Eng. C5 (1997) 153.

[4] C. Goubault, P. Jop, M. Fermigier, J. Baudry, E. Bertrand and J. Bibette Phys. Rev. Lett. 91 (2003) 260802.

[5] S.L. Biswal and A.P. Gast Analytical Chem. 76 (2004) 6448.

[6] K. Erglis, V. Ose, A. Zeltins and A. Cebers Magnetohydrodyn. 46 (2010) 23.

[7] C. Wilhelm, J. Browaeys, A. Ponton and J.C. Bacri Phys. Rev. E 67 (2003) 011504

[8] K. Erglis, D. Zhulenkovs, A. Sharipo and A. Cebers J. Phys.: Condens. Matter 20 (2008) 204107.

[9] O. Sandre, R. Perzynski, J.C. Bacri, V. Cabuil and R. Rosensweig Phys. Rev. E 59 (1999) 1736.

[10] J. Fresnais, J.-F. Berret, B. Frka-Petesic, O. Sandre and R. Perzynski Adv. Materials. 20 (2008) 3877.

[11] J. Fresnais, J.-F. Berret, B. Frka-Petesic, O. Sandre and R. Perzynski J. Phys.: Condens. Matter 20 (2008) 494216.

[12] P. Tierno, J. Claret, F. Sagues and A. Cebers Phys. Rev. E 79 (2009) 021501.

[13] R. Massart IEEE Trans. Magn. MAG 17 (1981) 1247.

[14] D. Taton, A.-Z. Wilczewska, M. Destarac Macromol. Rapid Comm. 22 (2001) 1497.

[15] F. Gazeau, F. Boué, E. Dubois and R. Perzynski J. Phys.: Condens. Matter 15 (2003) S1305.

[16] A. Anguelouch, R.L. Leheny, D.H. Reich Appl. Phys. Lett. 89 (2006) 111914.

[17] http://rsbweb.nih.gov/ij/

[18] K. Erglis, Qi Wen, V. Ose, A. Zeltins, A. Sharipo, P.A.Janmey and A. Cebers Biophysical J. 93 (2007) 1402.

[19] G. Helgesen, P. Pieranski and A.T. Skjeltorp Phys. Rev. Lett. 64 (1990) 1425.

[20] G. Helgesen, P. Pieranski and A.T. Skjeltorp Phys. Rev. A 42 (1990) 7271. 